\title{
Transfer of Learned Manipulation following Changes in Degrees of Freedom
}

\author{
Qiushi Fu, ${ }^{1}$ Ziaul Hasan, ${ }^{2}$ and Marco Santello ${ }^{1}$ \\ ${ }^{1}$ School of Biological and Health Systems Engineering, Arizona State University, Tempe, Arizona 85287, and ${ }^{2}$ Department of Kinesiology and Nutrition, \\ University of Illinois at Chicago, Chicago, Illinois 60612
}

The present study was designed to determine whether manipulation learned with a set of digits can be transferred to grips involving a different number of digits, and possible mechanisms underlying such transfer. The goal of the task was to exert a torque and vertical forces on a visually symmetrical object at object lift onset to balance the external torque caused by asymmetrical mass distribution. Subjects learned this manipulation through consecutive practice using one grip type (two or three digits), after which they performed the same task but with another grip type (e.g., after adding or removing one digit, respectively). Subjects were able to switch grip type without compromising the behavioral outcome (i.e., the direction, timing, and magnitude of the torque exerted on the object was unchanged), despite the use of significantly different digit force-position coordination patterns in the two grip types. Our results support the transfer of learning for anticipatory control of manipulation and indicate that the CNS forms an internal model of the manipulation task independent of the effectors that are used to learn it. We propose that sensory information about the new digit placement-resulting from adding or removing a digit immediately after the switch in grip type-plays an important role in the accurate modulation of new digit force distributions. We discuss our results in relation to studies of manipulation reporting lack of learning transfer and propose a theoretical framework that accounts for failure or success of motor learning generalization.

\section{Introduction}

Neural control of object manipulation is a topic of considerable interest. One paradigm that has been found useful for such studies involves lifting an object whose weight distribution is asymmetric, thus requiring the digits to exert not only a net vertical force but also a torque to prevent rolling of the object (Goodwin et al., 1998; Salimi et al., 2000, 2003; Lukos et al., 2007, 2008). Presented with this task, subjects learn to modulate digit forces as a function of digit placement to exert a compensatory torque in an anticipatory fashion (i.e., before lifting the object) (Fu et al., 2010).

To further understand the sensorimotor mechanisms underlying learning of manipulation, Zhang et al. (2010) examined subjects' ability to transfer learned digit force-position relations following an object rotation. Learning did not transfer readily as subjects failed to mentally rotate the learned action (i.e., the direction of compensatory torque) in response to the new object center of mass location. Learning of compensatory torque also

Received March 4, 2011; revised May 2, 2011; accepted July 27, 2011.

Author contributions: Q.F., Z.H., and M.S. designed research; Q.F. performed research; Q.F., Z.H., and M.S. analyzed data; Q.F., Z.H., and M.S. wrote the paper.

This publication was made possible by Bioengineering Research Partnership Grant R01-NS050265 from the National Institute of Neurological Disorders and Stroke (NINDS) at the National Institutes of Health (NIH) and Collaborative Research Grant IIS-0904504 from the National Science Foundation (NSF). Its contents are solely the responsibility of the authors and do not necessarily represent the official views of NIH, NINDS, or NSF. We thank the Neural Control of Movement Laboratory members for their comments on an earlier version of the manuscript.

Correspondence should be addressed to Marco Santello, School of Biological and Health Systems Engineering, 501 East Tyler Mall, ECG Building, Suite 334, Arizona State University, Tempe, AZ 85287-9709. E-mail: marco.santello@asu.edu.

DOI:10.1523/JNEUROSCI.1143-11.2011

Copyright $\odot 2011$ the authors $\quad 0270-6474 / 11 / 3113576-09 \$ 15.00 / 0$ does not transfer from one hand to another (Bursztyn and Flanagan, 2008; Jesunathadas et al., 2010). These results suggest that learning of manipulative actions had occurred in a hand, rather than an object, frame of reference. However, the factors that constrain the extent to which learned digit forces and positions can be transferred are not well understood. The present study was designed to determine whether manipulation learned with a set of digits can be transferred to grips involving a different number of digits.

The ability to perform the same behavioral task using different sensorimotor elements is a manifestation of "motor equivalence" - a phenomenon remarked upon in the early history of motor control (Lashley, 1930; Bernstein, 1967)—but studied only sporadically. The problem of motor equivalence can be defined as follows: are the neural representations of actions specific to, and constrained by, the effectors (e.g., muscles, limbs) used to learn a given action, hence performable only with the same effectors, or are they independent from "how" they were learned? Changing the number of fingers that participate in the lifting task allows us to address this question. An important characteristic of our experimental protocol is that transfer of the compensatory torque across grip types can be implemented through an infinite number of new relations between the control variables: grip force, difference in the lift force between the two sides, and vertical distance between contact points on the two sides. We hypothesized that subjects will be able to transfer the compensatory torque on the first trial following a change in the number of fingers, although the combinations of digit forces and positions do not remain the same as those learned before the change in grip type. 
A

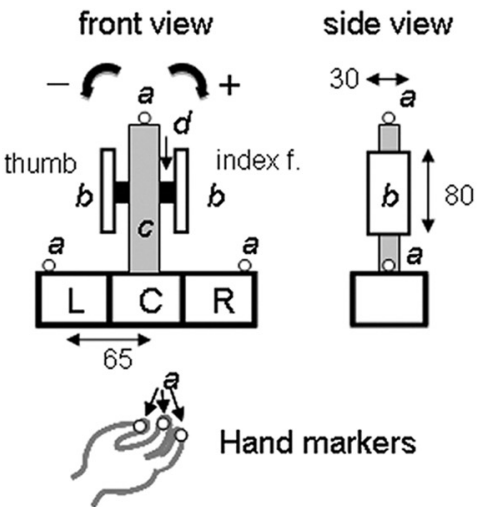

B

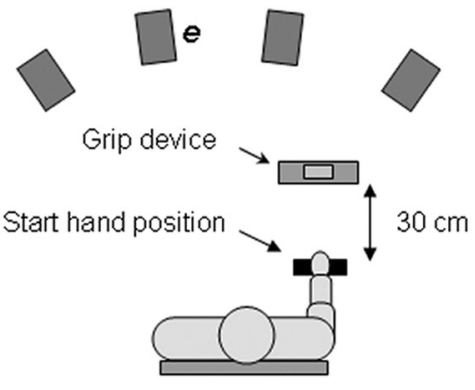

C

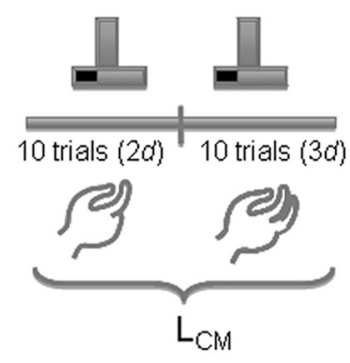

Session \#1: 2d $\rightarrow 3 d$

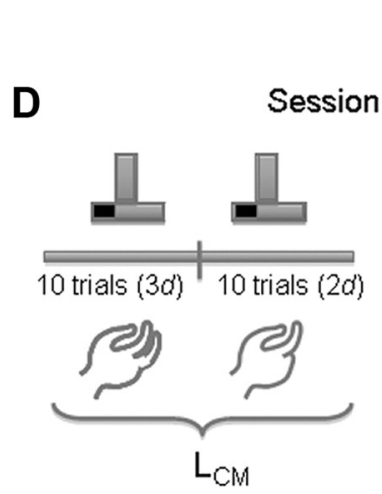

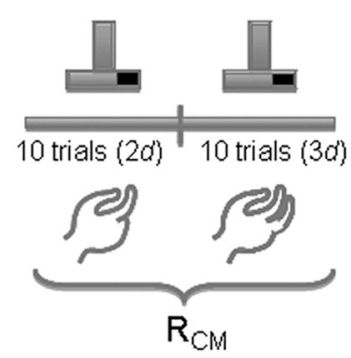

2 weeks

Figure 1. Experimental setup and procedures. $\boldsymbol{A}$, Front and side views of the grip device used to measure forces and centers of pressure on the grasp surfaces for the thumb and finger sides (units are in millimeters). Object position and orientation were tracked through a motion capture system and active markers (denoted as small spheres, $a$ ) were placed on the top and on the extremities of the bottom box of the grip device. Active markers were also placed on the nails of the thumb, index, and middle fingers. A mass ( $400 \mathrm{~g}$ ) was inserted in either the left ( $\mathrm{L}$ ) or right ( $\mathrm{R}$ ) compartment in the bottom box of the device to change the center of mass of the object ( $\mathrm{L}_{\mathrm{CM}}$ and $\mathrm{R}_{\mathrm{CM}}$ conditions, respectively). The convention for defining the direction of object roll (negative and positive toward the thumb or finger side, respectively) is also shown. The configuration of the grip device consisted of a central block ( $c$ ) and two bars (grip surfaces, $b$ ), each mounted on a force/torque sensor ( $d$ ). $\boldsymbol{B}$, Top view of the experimental procedures. Subjects reached to the grip device located at $30 \mathrm{~cm}$ from the start position. Infrared cameras (e) were placed around the workspace to track hand and object kinematics. C, D, Trial sequences associated with switching from two to three digits $(2 d \rightarrow 3 d)$ and vice versa $(3 d \rightarrow 2 d)$, respectively. Subjects were tested on experimental session 2,2 weeks after experimental session 1 . All subjects started each experimental session with the $\mathrm{L}_{\mathrm{CM}}$ using each grip type, followed by an equal number of trials with a different grip type and the $\mathrm{R}_{\mathrm{CM}}$.

\section{Materials and Methods}

Subjects. Ten right-handed volunteers (4 males and 6 females, mean age and SD: $21.4 \pm 2.2$ years) participated in this study. Subjects had no previous history of orthopedic, neurological trauma, or pathology of the upper limbs and were naive to the purpose of the study. Subjects gave their informed consent according to the declaration of Helsinki and the protocols were approved by the Office of Research Integrity and Assurance at Arizona State University.

Apparatus. We used a custom-made grip device to measure digit forces and their points of application (Fig. $1 \mathrm{~A}$ ) (for details, see Fu et al., 2010). Briefly, two six-component force/torque (F/T) transducers (Nano-17, ATI Industrial Automation; nominal force resolution: $0.012 \mathrm{~N}$; nominal torque resolution $0.63 \mathrm{~N} \mathrm{~cm}$ ) (Fig. $1 \mathrm{~A}, \mathrm{~d}$ ) mounted collinear to each other on the grip device recorded forces and torques exerted by the thumb on one side and fingers on the other. The grip surfaces consisted of two parallel polyvinyl chloride plates (Fig. 1 A, b) each mounted vertically on an F/T transducer and were covered with 100 grit sandpaper (static friction coefficients: 1.4-1.5). The distance between the two grip surfaces (grip width) was $6.07 \mathrm{~cm}$.

A Plexiglas box attached underneath the grip apparatus was used to change the mass distribution to the left or right of the grip device midline by inserting a mass $(400 \mathrm{~g})$ into one of three compartments (Fig. $1 A$ ). The total mass of the grip device and load was $790 \mathrm{~g}$. When the load was placed in the left or right compartment (Fig. $1 \mathrm{~A}, \mathrm{~L}$ and $\mathrm{R}$ ), it introduced a torque on the frontal plane of -255 and $+255 \mathrm{~N} / \mathrm{mm}$, respectively. View of the added mass location was blocked by a lid to prevent visual identification of the object center of mass (CM).

We recorded hand and object kinematics using an active marker 3D motion capture system (PhaseSpace; frame rate: $480 \mathrm{~Hz}$; spatial accuracy: $\sim 1 \mathrm{~mm}$; spatial resolution: $0.1 \mathrm{~mm}$ ) with eight cameras (Fig. $1 B, \mathrm{e}$ ). Subjects were outfitted with light-weight visible red emitting diode
(RED) active markers ( $5 \mathrm{~mm}$ in diameter) on the fingernails of thumb, index, and middle fingers. Markers were also placed on the lateral extremities of the object and on its top to track its position and orientation during the lift (Fig. $1 \mathrm{~A}$, a). Before data collection, we verified that placement of the RED markers did not prevent motion of the digits and/or the wrist by asking subjects to fully flex and extend all digits as well as to grasp the object before the start of the experiment. Force and torque data were acquired by 12 bit analog-to-digital converter boards (PCI-6225, National Instrument; sampling frequency: $1 \mathrm{kHz}$ ). Data acquisition was performed through LabView (version 8.0, National Instrument).

Experimental procedures. Subjects were asked to sit facing the grip device (Fig. $1 B$ ) with the elbow flexed at $\sim 70-90^{\circ}$ in the parasagittal plane, to align their right shoulder with the midpoint of the grip device, and to place their hand (palm facing downward) on a support located 30 $\mathrm{cm}$ from the grip device. After a verbal signal from the experimenter, subjects reached from this start location, grasped the grip surfaces with the tip of either the thumb and index finger or thumb, index, and middle fingers of the right hand, lifted the grip device at a natural speed to a height of $\sim 10 \mathrm{~cm}$, held it for $\sim 1 \mathrm{~s}$, and replaced it to its start location. We asked subjects to extend the noninvolved fingers throughout the task to ensure that only the tip of the thumb and index finger (or thumb, index, and middle fingers) contacted the grip surfaces. Compliance was visually verified on each trial by one of the experimenters. At the beginning of each block of trials, we instructed subjects to minimize object roll during the lift. The experimenter gave no instructions about where to grasp the object, thus leaving subjects the choice of digit placement anywhere along the grip surface (Fig. $1 A, b$ ) to comply with the requirement of minimizing object roll.

Each subject performed the task under the following two experimental conditions: (1) two-digit grasping (thumb and index finger; $2 d$ ) and (2) three-digit grasping (thumb, index, and middle fingers; $3 d$ ). On the first 
experimental session, subjects performed $102 d$ trials followed by $103 d$ trials $(2 d \rightarrow 3 d)$ on the left $\mathrm{CM}\left(\mathrm{L}_{\mathrm{CM}}\right)$ condition (Fig. 1C). The between-trial interval within a block of 10 trials was $\sim 10 \mathrm{~s}$. After a short break $(\sim 20 \mathrm{~s})$, subjects performed the $2 d \rightarrow 3 d$ experimental condition on the right $\mathrm{CM}\left(\mathrm{R}_{\mathrm{CM}}\right)$ condition. Each subject was tested again 2 weeks later but on a trial sequence opposite to that experienced on his/her first experimental session (i.e., $3 d$ $\rightarrow 2 d$ on the $\mathrm{L}_{\mathrm{CM}}$ condition followed by $3 d \rightarrow$ $2 d$ on the $\mathrm{R}_{\mathrm{CM}}$ condition) (Fig. $1 D$ ). This design was motivated by the need to examine whether learning transfer of the compensatory torque following a switch from $2 d$ to $3 d$ is equivalent to switching from $3 d$ to $2 d$. The break between the two experimental sessions was used to minimize potential positive or negative learning transfer effects from one sequence to the next. The effectiveness of the 2 week break in preventing positive or negative learning transfer was confirmed statistically.

Before the experiment, we asked subjects to lift the object once with each hand configuration $(2 d$ and $3 d)$ with the load placed in the center compartment (Fig. $1 \mathrm{~A}, \mathrm{C}$ ) to themselves familiarize with the task, texture, and weight of the grip device. Thereafter and at the beginning of each block of trials, we informed subjects that (1) the load could be placed either in the left or right compartment of the Plexiglas box ( $\mathrm{L}_{\mathrm{CM}}$ and $\mathrm{R}_{\mathrm{CM}}$, respectively), (2) it would remain the same for a block of 10 consecutive trials, and (3) the number of digits to be used for the upcoming block of trials. After the subject performed 10 consecutive trials with a given grip type for the $\mathrm{L}_{\mathrm{CM}}$ condition, we told the subject to perform another block of 10 consecutive trials but with a different number of digits. We emphasized that the goal of the task remained the same, that is, to minimize object roll during the lift. After the subject performed a total of 20 trials ( 10 with each grip type), subjects were informed that the object CM would be changed, and that they will be asked to perform two more blocks of 10 trials each for each grip type. Before subjects started the $\mathrm{R}_{\mathrm{CM}}$ block of trials, we repeated the same instructions given for the $\mathrm{L}_{\mathrm{CM}}$ block of trials. Throughout the experiment, we blocked the view of placement of the mass in the left or right compartment of the object to prevent subjects from anticipating the new CM location on the first object lift.

Data processing. Force and position data were temporally aligned offline and analyses were performed using MATLAB. We analyzed the following variables (Fig. 2) (for details, see Fu et al., 2010): (1) object lift onset, the time at which the vertical position of the grip device crossed and remained above a threshold for $200 \mathrm{~ms}$; (2) object roll, the angle between the gravitational vector and the vertical axis of the grip device, and peak roll, the peak of object roll shortly $(\sim 150 \mathrm{~ms})$ after object lift onset; (3) digit forces, force perpendicular [grip force (GF)] and parallel [load force (LF)] to the grip surface; and (4) digit center of pressure $(\mathrm{CoP})$, the vertical coordinate of the point of resultant digit force application, calculated for each digit using the force and torque output of each sensor (positive and negative values for CoP denoted higher and lower $\mathrm{CoP}$ values relative to the center of transducer, respectively). Note that GF, LF, and CoP recorded on the finger side of the grip device are the resultant net forces and net center of pressure of both index and middle finger when subjects performed the task using the $3 d$ grip. To quantify the modulation of individual digit position, we recorded fingertip marker position, defined as the vertical position of the marker on the nail of the thumb, index, and middle fingers.
We used digit forces and CoP to compute the following performance variables: (1) the average of the digit grip force $\left(F_{\mathrm{GF}}\right)$ values; $(2)$ the difference between load forces exerted on the thumb and finger side of the grip device $\left(d_{\mathrm{LF}}\right)$; and (3) the vertical distance between the CoP on the thumb and finger side of the grip device $\left(d_{y}\right)$. These three variables are important to produce the compensatory torque $\left(T_{\mathrm{com}}\right)$ for minimizing object roll (i.e., balancing the external torque caused by the added mass) (for details, see Fu et al., 2010). To further understand how digit placement changed following a change in grip type, we also computed the vertical distance between thumb and index finger markers $\left(d_{\text {tip }}\right)$. Note that all of these performance variables were computed at object lift onset.

Learning and learning transfer of compensatory torque. Our previous work has shown that subjects learn to generate $T_{\text {com }}$ in an anticipatory fashion (i.e., at object lift onset) within the first three consecutive object lifts (Fu et al., 2010; Zhang et al., 2010). Here we quantified again the trial-to-trial learning of $T_{\text {com }}$ with a given grip type, $2 d$ or $3 d$, as an intermediate step to test subjects' ability to generate the same $T_{\text {com }}$ after changing grip type to $3 d$ or $2 d$, respectively. For learning transfer to be defined as positive, $T_{\text {com }}$ generated before the grip type switch had to be statistically indistinguishable from $T_{\text {com }}$ generated on the first trial (trial 11) following the switch. For our study, a positive learning transfer of $T_{\text {com }}$ would be evidence for motor equivalence, as the same global variable is generated in an effector-independent fashion (e.g., regardless of the number of digits used to lift the object). Conversely, statistically significant differences in $T_{\text {com }}$ preswitch versus postswitch in grip type would suggest a failure of learning transfer, which could result from the following three different phenomena: (1) same $T_{\text {com }}$ on trial 1 and 11, indicating a "reset" of subjects' learned behavior to the same state asso- 
ciated with the very first trial with the same object CM (no transfer); and significantly, (2) larger or (3) smaller $T_{\text {com }}$ on trial 11 than on trial 10, indicating an anterograde effect of the learned $T_{\text {com }}$ with a given grip type on $T_{\text {com }}$ with a different grip type. A larger than necessary $T_{\text {com }}$ may result in an overcompensation of the external torque, hence generating a roll in the opposite direction to that caused by the added mass, whereas a smaller than necessary $T_{\text {com }}$ would be insufficient to prevent object roll to the same extent attained up to the switch to a different grip type. Both of these instances can be defined as partial transfer, as they maintain some features of the learned behavior that, at the same time, are functionally better than the initial state associated with trial 1 .

Although on a trial-to-trial basis subjects could theoretically use different relations among $d_{y}, d_{\mathrm{LF}}$, and $F_{\mathrm{GF}}$ to generate a given $T_{\text {com }}$, after the first few lifts each subject tends to use the same relation consistently when performing consecutive object lifts (Fu et al., 2010). Here we address the question of how subjects transfer the learned relation following a change in grip type. For example, a positive transfer of $T_{\text {com }}$ on trial 11 might occur through the adoption of the same relation between its three components used before the grip type switch or, alternatively, by changing the relation such that, for example, subjects choose to exert different $d_{\mathrm{LF}}$ through different $d_{y}$. This example shows that, to fully understand learning transfer of $T_{\text {com }}$, it is necessary to examine how the individual $T_{\text {com }}$ components were transferred across grip types.

Statistical analysis. To quantify learning through consecutive object lifts, we performed ANOVA with repeated measures within the preswitch blocks of 10 trials for each CM location on $T_{\text {com }}$ using trial (10 levels) as the within-subject factor. The goal of these analyses was to test whether learning of $T_{\text {com }}$ had occurred when using a given grip type on a block of consecutive trials before switching to a different grip type. Our previous work (Fu et al., 2010; Zhang et al., 2010) revealed that subjects learn to generate consistent $T_{\text {com }}$ after the third trial. We verified this in the present study by performing ANOVA with repeated measures for each $\mathrm{CM}$ location with within-subject factors of trial (4 through 10 of the trial block before the switch in grip type) (Fig. 3). Lack of significant main effect of trial would indicate that subjects generated a stable $T_{\text {com }}$ throughout the last seven trials of the preswitch block. Transfer of $T_{\text {com }}$ and modulation of its components $\left(F_{\mathrm{GF}}, d_{\mathrm{LF}}\right.$, and $\left.d_{y}\right)$ was quantified using ANOVA with repeated measures in the following two ways: (1) as the immediate transfer and modulation, quantified by comparing the last trial before the switch in grip type versus the first trial following the switch; and (2) as long-term transfer and modulation, quantified by the average difference between preswitch versus postswitch trials. Sphericity assumptions were tested for all analyses (Greenhouse-Geisser analysis), and the results were corrected when appropriate. All tests were performed at the $p \leq 0.05$ significance level.

\section{Results}

We describe the results in the following three sections: (1) learning and learning transfer of $T_{\text {com }}$; (2) immediate modulation of $T_{\text {com }}$ components on the trial following the switch in grip type; and (3) long-term adaptation of $T_{\text {com }}$ components throughout consecutive trials following the switch in grip type.

\section{Learning and learning transfer of compensatory torque}

Figure 2 shows the time course of $T_{\text {com }}$ (top row, dashed line) and its components on the first and last trial performed using a twodigit grip, and data from the first trial performed with a threedigit grip by a representative subject. On trial 1, the subject does not know the CM location (left CM), and therefore no $T_{\text {com }}$ is generated at object lift onset (Fig. 2, vertical solid line). As a result, the object rolls toward the thumb $\sim 20^{\circ}$ during the lift due to the external torque caused by the added mass before a corrective response can be initiated. By trial 10, however, this subject generated $T_{\text {com }}$ of magnitude and direction appropriate to minimize object roll during the lift. The appropriate $T_{\text {com }}$ was generated through a concurrent modulation of digit load forces and center of pressure. Specifically, at object lift onset this subject
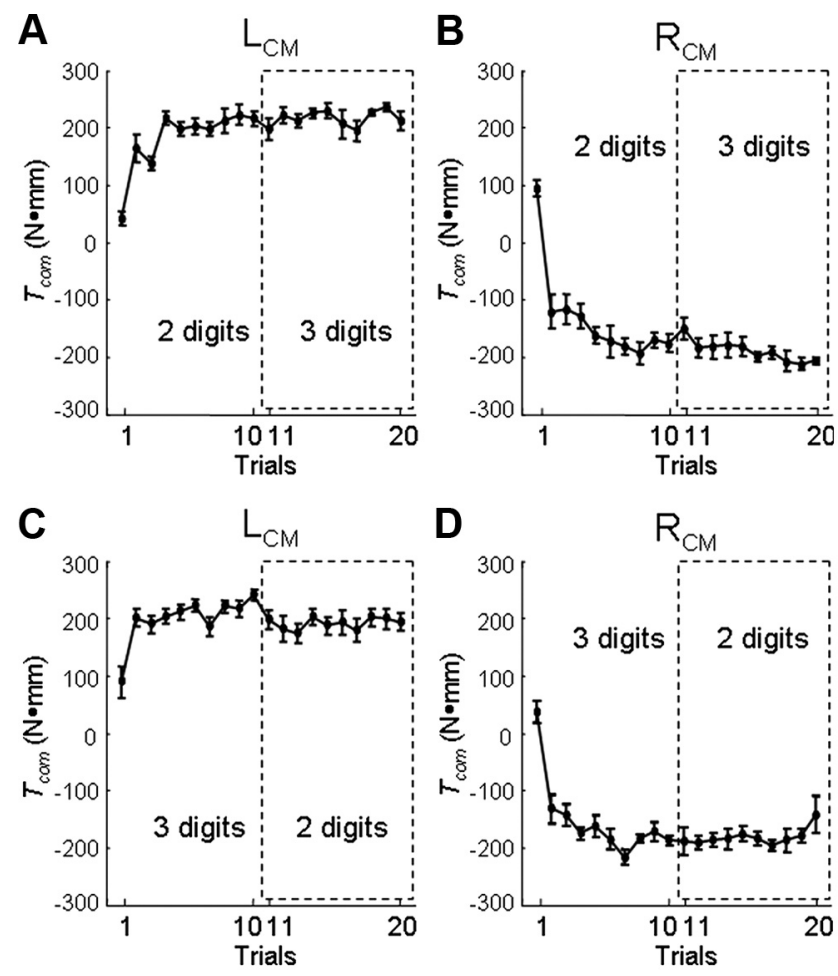

Figure 3. Learning curves of compensatory torque: pregrip and postgrip type switch. $A, B$ $T_{\text {com }}$ for two-digit grip trials followed by three-digit grip trials for left and right CM conditions $(\boldsymbol{A}$, $\mathrm{L}_{\mathrm{CM}} ;$ and $\left.\boldsymbol{B}, \mathrm{R}_{\mathrm{CM}}\right) . \boldsymbol{C}, \boldsymbol{D}, T_{\text {com }}$ data from grip type presented in the reverse order (three-digit grip followed by two-digit grip) for $\mathrm{L}_{C M}(\boldsymbol{C})$ and $\mathrm{R}_{\mathrm{CM}}(\boldsymbol{D})$. Trials within the dashed box (11-20) indicate the grip type subjects switched to after learning $T_{\text {com }}$ during consecutive object lifts (trials 1-10) with a different grip type. Data are the averages of all subjects (vertical bars denote SES).

positioned the thumb higher than the index finger (positive $d_{y}$ ), exerted a larger thumb load force (positive $d_{\mathrm{LF}}$ ), and a slightly larger grip force $\left(F_{\mathrm{GF}}\right)$ relative to trial 1. Consistent with previous observations (Fu et al., 2010; Zhang et al., 2010), $T_{\text {com }}$ averaged across all subjects changed significantly as a function of consecutive practice in the preswitch block (significant main effect of trial; $2 d, \mathrm{~L}_{\mathrm{CM}}: F_{(1,9)}=17.31 ; 2 d, \mathrm{R}_{\mathrm{CM}}: F_{(1,9)}=26.85 ; 3 d, \mathrm{~L}_{\mathrm{CM}}$ : $F_{(1,9)}=12.04 ; 3 d, \mathrm{R}_{\mathrm{CM}}: F_{(1,9)}=25.12$; all $p<0.001$ ) (Fig. 3). On average, all subjects learned to anticipate the $T_{\text {com }}$ necessary to minimize object roll within the first three trials regardless of grip type and CM location (trials 1-3, Fig. 3 ), after which $T_{\text {com }}$ did not change any further on subsequent trials (all tests on trials 4-10: $p>0.05)$.

Remarkably, following a change in grip type (trial 11), the subject in Figure 2 maintained the ability to generate a similar $T_{\text {com }}$ to that generated on trial 10 , hence equally appropriate in minimizing object roll. However, on trial 11 this subject used a different relation among $d_{y}, d_{\mathrm{LF}}$, and $F_{\mathrm{GF}}$ from that used on trial 10. Specifically, forcing a subject to add the middle finger resulted in the application of a slightly larger grip force, less asymmetrical sharing of load forces, and positioning of the index finger to a higher position. Furthermore, the index finger was positioned lower than the thumb in trial 10 but higher than the thumb in trial 11 , and the middle finger was positioned lower than the thumb. The major normal force contribution on the finger side is shifted from the index finger to the middle finger as indicated by the lowering of net center pressure closer to the middle finger position. Note that these 
Table 1. Statistical results on immediate transfer of action and adaptation of compensatory torque components (trial 10 vs 11$)$

\begin{tabular}{llll}
\hline & $2 d \rightarrow 3 d \mathrm{~L}_{\mathrm{CM}}$ & $2 d \rightarrow 3 d \mathrm{R}_{\mathrm{CM}}$ & $3 d \rightarrow 2 d \mathrm{~L}_{\mathrm{CM}}$ \\
\hline$T_{\text {com }}$ & n.s. & n.s. & \multicolumn{1}{c}{$3 d \rightarrow 2 d \mathrm{R}_{\mathrm{CM}}$} \\
Peak roll & n.s. & n.s. & n.s. \\
$d_{y}$ & $F=8.13, p=0.019$ & $F=20.05, p=0.002$ & n.s. \\
$d_{\mathrm{LF}}$ & $F=14.9, p=0.004$ & n.s. & n.s. \\
$F_{\mathrm{GF}}$ & n.s. & n.s. & n.s. \\
$d_{\text {tip }}$ & $F=16.30, p=0.003$ & $F=15.40, p=0.035$ & n.s. \\
\hline
\end{tabular}

Each row shows statistical results of simple effects of switching grip type in two $C M$ conditions $\left(L_{C M}\right.$ and $R_{C M}$ ) on a single variable, compared between trial 10 (preswitch trial) and trial 11 (postswitch trial). The degrees of freedom for all comparisons are (1,9). n.s., Nonsignificant.

changes all occur since early contact, suggesting anticipatory planning for new grip type.

We performed ANOVA for each CM location with within-subject factor of grip type (two levels; $2 d$ and $3 d$ ) to examine the immediate effect of changing grip type on $T_{\text {com}}$. When subjects changed grip type, on the very first trial (trial 11, Fig. 3) they were able to generate $T_{\text {com }}$ whose magnitude was statistically indistinguishable from that generated on the preswitch trial (Fig. 3, trial 10) for all but one experimental condition ( $3 d \rightarrow 2 d, \mathrm{~L}_{\mathrm{CM}}$; Table 1$)$. However, no significant differences were found when comparing peak object roll on trial 10 versus trial 11 on any of the four experimental conditions. This indicates that the statistically significant difference for the $3 d \rightarrow 2 d \mathrm{~L}_{\mathrm{CM}}$ condition did not have significant behavioral consequences on the manipulation, thus suggesting that anticipatory control of $T_{\text {com }}$ in the preswitch and postswitch trials was equally appropriate to attain the task goal.

To further quantify the extent to which $T_{\text {com }}$ was transferred from one grip type to another, we examined average differences between seven trials preswitch versus postswitch in grip type by ANOVA with repeated measures for each CM location with within-subject factors of trial (seven levels; seven preswitch and seven postswitch) and grip type (two levels, preswitch and postswitch). We found no significant main effect of trial, grip type, or interaction $(p>0.05$ for each experimental condition). This suggests that no further learning of $T_{\text {com }}$ occurred before and after the switch in grip type, the average $T_{\text {com }}$ being statistically similar for the two grip types. As expected from the results on $T_{\text {com }}$, we found no significant main effect of trial, grip type, or interaction on peak object roll $(p>0.05$ for each experimental condition). Therefore, the significant difference in $T_{\text {com }}$ preswitch versus postswitch trial in $3 d \rightarrow 2 d \mathrm{~L}_{\mathrm{CM}}$ condition could have been caused by $\mathrm{CM}$-specific and hand posturespecific control strategies. With regard to digit position modulation, two-digit grip with the left CM may challenge the modulation of $d_{y}$, hence $T_{\text {com }}$, differently than a three-digit grip. For the left CM condition, subjects can adopt a larger $d_{y}$ when grasping the object with three rather than two digits because, in the former case, the middle finger can be positioned lower than index finger, thus further shifting the net CoP on the finger side of the object. However, for the right CM condition two-digit grip allows subjects to use a larger $d_{y}$ than three-digit grip because the net $\mathrm{CoP}$ on the finger side will always be higher when force is
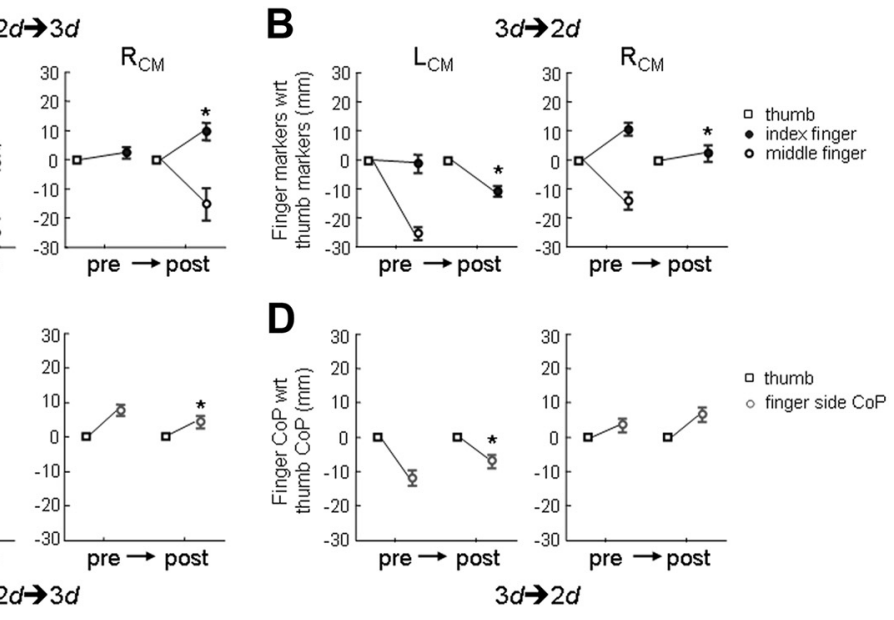

$2 d \rightarrow 3 d$

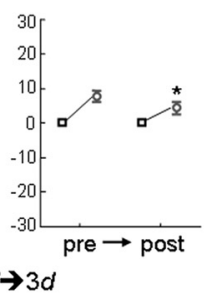

Figure 4. Learning transfer of digit center of pressure and position from pregrip to postgrip type switch trials (immediate transfer). Top panels show the thumb, index, and middle fingertip position defined by the vertical marker position. To visualize the relative position of the fingertips, the thumb marker position (square) is connected by a line to the index and middle finger marke to two digits). Asterisks denote a statistically significant difference $(p<0.05)$ between preswitch and postswitch trials. Data are

exerted only with the index finger than when force is exerted by both index and middle fingers. With regard to force modulation, index and middle fingers share the load in three-digit grip while index has to sustain by itself most of the load on finger side in two-digit grip. Because of the above differences in digit position and force modulation, two-digit grip for left CM requires subjects to use only the index finger to sustain a relatively larger load than three-digit grip. This might have caused the significant change in $T_{\text {com }}$ for the $3 d \rightarrow 2 d \mathrm{~L}_{\mathrm{CM}}$ condition. Nevertheless, and most importantly, the overall task performance (minimizing object roll) was not degraded by switching grip type.

\section{Immediate adaptation of compensatory torque components following a change in grip type}

The above positive learning transfer of $T_{\text {com }}$ to a different grip type implies that subjects were able to coordinate, in an anticipatory fashion, the three $T_{\text {com }}$ components, $d_{y}, d_{\mathrm{LF}}$, and $F_{\mathrm{GF}}$. However, these three components can be coordinated in an infinite number of ways. Therefore, to determine the solutions chosen by subjects to generate the same $T_{\text {com }}$ after changing grip type, we analyzed each $T_{\text {com }}$ component separately. The analyses below addressed the question of whether subjects chose the same or different compensatory torque components immediately following a change in the number of fingers participating to the grasp using ANOVA for each CM location with within-subject factor of grip type (two levels; $2 d$ and $3 d$ ). 
Digit center of pressure

Subjects used significantly different vertical separations between digit center of pressure $\left(d_{\gamma}\right)$ after switching grip type on all but one experimental condition $\left(3 d \rightarrow 2 d, \mathrm{R}_{\mathrm{CM}}\right)$ (Fig. $4 D$; Table 1 ). For the left CM location, subjects significantly increased $d_{y}$ when adding the middle finger to the grip (Fig. 4C) and decreased $d_{y}$ when removing one finger from the grip (Fig. 4D), whereas an opposite pattern was found for the right CM location. However, the change in the net center of pressure on the finger side when adding or removing a finger could have been due to (1) a change in force distribution while keeping the original distance between thumb and index finger the same or (2) to a change in the index finger position relative to thumb position and force distribution. To distinguish between these two possibilities, we tracked the thumb, index, and middle fingertip position through a motion capture system. We found that the $d_{\text {tip }}$ was significantly modulated such that the index finger was positioned higher (Fig. 4A) when adding the middle finger and lower when removing the middle finger (Fig. 4B). Therefore, subjects used significantly different digit placement distribution when changing grip type (Table 1).

\section{Digit grip force}

We found no significant main effect of grip type $(p>0.05$ for each experimental condition), indicating that subjects exerted similar net grip forces regardless of the number of digits used for the grasp (Table 1). However, while grip force is provided by index finger only in $2 d$ grip on finger side, the middle finger may contribute the substantially in $3 d$ grip for left CM condition.

\section{Digit load force}

Subjects used significantly different load force sharing (thumb minus finger load force; $d_{\mathrm{LF}}$ ) after switching grip type on only one experimental condition $\left(2 d \rightarrow 3 d, \mathrm{~L}_{\mathrm{CM}}\right.$; Table 1$)$.

In summary, the immediate effects of changing grip type were mostly found on grasp kinematics, as the overall force coordination was little affected by adding or removing the middle finger. This indicates that, since subjects generated similar $T_{\text {com }}$ on preswitch versus postswitch in grip type, significant changes in digit placement were actively compensated by redistribution of digit forces. Although we cannot explicitly measure individual grip forces of the index and middle fingers, the redistribution of grip force can be inferred from the location of the net CoP and fingertip positions. The fact that net $\mathrm{CoP}$ was located about halfway between the index and middle fingers indicates an approximately even sharing of grip forces.

\section{Long-term adaptation of compensatory torque components following a change in grip type}

The above analysis quantified subjects' immediate ability to generate the same $T_{\text {com }}$ on the trial before and after the switch in grip type. However, a complementary question is whether learning transfer effects might have gone beyond the very first trial performed with a different grip type. The analyses below were performed to quantify the extent to which $T_{\text {com }}$ learned with a given grip type had long-term effects on the trial-to-trial adaptation of digit forces and positions when performing object lifts using a different grip type. We performed ANOVA with repeated measures for each CM location with within-subject factors of trial (seven levels; seven preswitch and seven postswitch) and grip type (two levels, preswitch and postswitch).

\section{Digit center of pressure}

After the immediate adaptation (i.e., trial 11) following a change in grip type, there was no further significant modulation of $d_{y}$
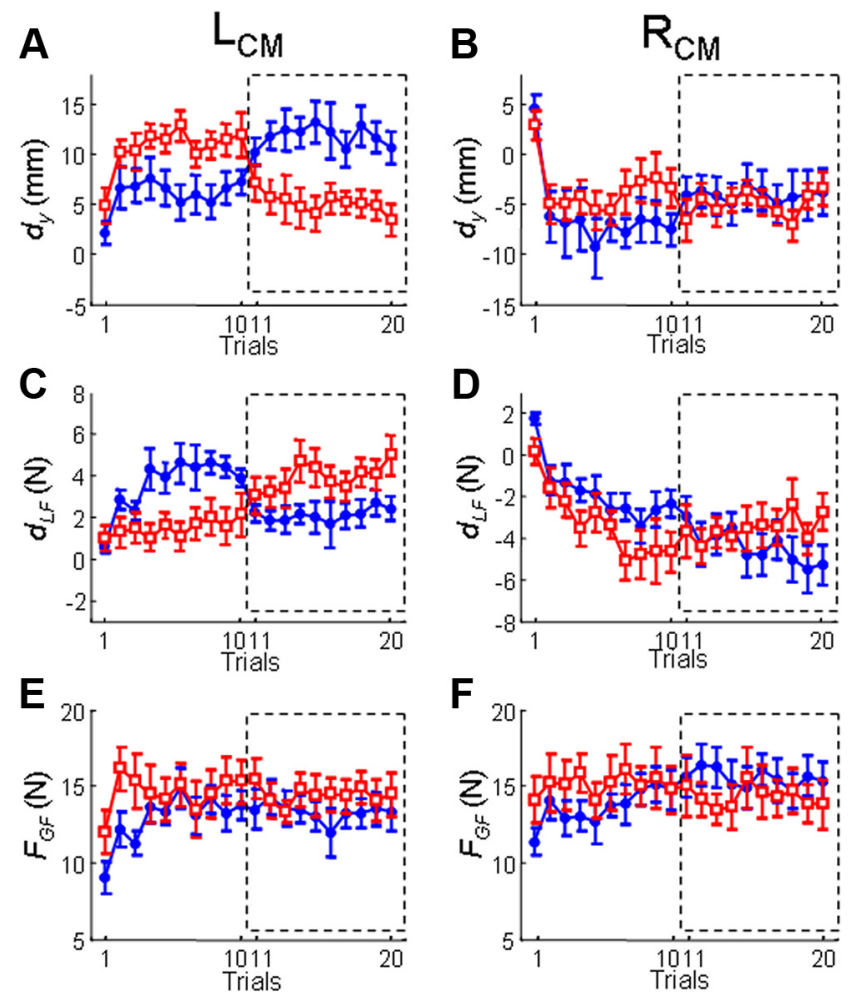

$$
2 d \rightarrow 3 d
$$

$3 d \rightarrow 2 d$

Figure 5. Learning curves of digit center of pressure and forces: pregrip and postgrip type switch (long-term adaptation). From top to bottom, data shown are the $d_{y^{\prime}}$ the $d_{\mathrm{LF}}$ and the $F_{\mathrm{GF}}$ respectively. For $3 d$, the center of pressure and forces exerted on the finger side of the device result from forces exerted by the index and middle fingers. Data on the left and right column are from the $L_{C M}$ and $R_{C M}$, respectively. Each plot shows data from two-digit grip trials followed by three-digit grip trials (squares) and trials performed in the reverse order (circles). Data are averages of all subjects ( $\pm \mathrm{SE}$ ).

(neither significant trial effect nor trial $\times$ grip interaction, $p>$ 0.05). Specifically, the new digit placement was maintained for all experimental conditions (significant grip type effect for three conditions and nonsignificant grip type effect for one condition, $3 d \rightarrow 2 d, \mathrm{R}_{\mathrm{CM}}$; this significant effect is consistent with the statistical significance of immediate adaptation) (Fig. 5; Table 2). With regard to the relative fingertip positions, $d_{\text {tip }}$ was significantly modulated in a similar fashion to that observed in the immediate adaptation in all conditions (significant grip type effect only; Table 2).

\section{Digit load forces}

Unlike the above-described immediate adaptation in the digit load force difference $\left(d_{\mathrm{LF}}\right)$, three of our experimental conditions showed long-term modulation of $d_{\mathrm{LF}}$ throughout the first postswitch trials in response to the modulation of $d_{y}$ (significant grip effect; Table 2$). d_{\mathrm{LF}}$ remained unchanged only in $3 d \rightarrow 2 d, \mathrm{R}_{\mathrm{CM}}$ condition (Table 2 ) in which $d_{y}$ also was not modulated significantly. However, there was neither a significant trial effect nor trial $\times$ grip type interaction (Fig. 5) on $d_{\mathrm{LF}}$. In general, subjects tend to use larger load force difference in $2 d$ grip than $3 d$ grip.

Digit grip force

In the trials following the switch in grip type, subjects exerted similar grip forces to those exerted before the switch (Fig. $5 E, F$ ), as indicated by the lack of significant main effects of grip type, 
Table 2. Statistical results on long-term transfer of action and adaptation of compensatory torque components (trials 4-10 vs 11-17)

\begin{tabular}{llll}
\hline & $2 d \rightarrow 3 d \mathrm{~L}_{\mathrm{CM}}$ & $2 d \rightarrow 3 d \mathrm{R}_{\mathrm{CM}}$ & $3 d \rightarrow 2 d \mathrm{~L}_{\mathrm{CM}}$ \\
\hline$T_{\text {com }}$ & n.s. & n.s. & n.s. \\
Peak roll & n.s. & n.s. & n.s. \\
$d_{y}$ & $F=41.84, p<0.001$ & $F=9.33, p=0.014$ & $F=20.98, p=0.001$ \\
$d_{\mathrm{LF}}$ & $F=22.07, p=0.001$ & $F=5.72, p=0.041$ & $\mathrm{n}=10.03, p=0.011$ \\
$F_{\mathrm{GF}}$ & n.s. & n.s. & n.s. \\
$d_{\text {tip }}$ & $F=15.43, p=0.003$ & $F=17.36, p=0.002$ & n.s. \\
\hline
\end{tabular}

Each row shows statistical results of simple effects of switching grip type in two $C M$ conditions ( $L_{C M}$ and $R_{C M}$ ) on a single variable, compared between trials 4-10 (preswitch trials) and trials $11-17$ (postswitch trials). The degrees of freedom for all comparisons are (1,9). n.s., Nonsignificant.

trial, or interaction $(p>0.05$ for each experimental condition; Table 2).

To summarize, whereas similar $T_{\text {com }}$ was generated over consecutive trials following a switch in grip type, subjects adopted different strategies in digit kinematics and forces. All three conditions that showed significant change in $d_{y}$ were compensated by significant change in load force coordination, whereas the $3 d \rightarrow$ $2 d, \mathrm{R}_{\mathrm{CM}}$ condition did not show any significant change except finger tip position. Lack of trial effects indicates that there was no further adaption beyond the immediate switch trial.

\section{Discussion}

After learning to lift an object with asymmetrical mass distribution using one grip type, subjects were able to immediately transfer the learned compensatory torque despite the addition or removal of one finger, thus preserving the ability to minimize object roll. This suggests that the manipulation learned through consecutive practice resulted in a high-level neural representation independent from low-level constraints (i.e., the specific effectors used to learn the manipulation). We discuss our results in relation to neural representations of manipulation tasks and the factors that limit their generalization.

\section{Motor equivalence in anticipatory control of manipulation}

The ability of the sensorimotor system to perform the same task using degrees of freedom that had not been engaged while learning the task has been referred to as motor equivalence (Lashley, 1930; Cole and Abbs, 1986; Rijntjes et al., 1999; Wing, 2000). Further examples of motor equivalence in anticipatory control of grasping are provided by across-hand transfer of object weight information (Chang et al., 2008). The present findings are evidence of within-hand motor equivalence that uses different fingers as different degrees of freedom. Of particular relevance to the present study are the observations that subjects can change multidigit force coordination patterns after removing or adding one finger to the grip during object hold with no disruption to the task or its mechanical requirements (Santello and Soechting, 2000; Budgeon et al., 2008). These studies showed that motor output can be reorganized within hand while performing the task with online sensing of the desired manipulation goal. The present findings extend these observations to anticipatory learning transfer. It appears that learned sensorimotor representation of a manipulation task can be used as a reference to which incoming sensory inputs can be compared for generating behaviorally equivalent outputs through different degrees of freedom (e.g., using of different fingers). This is an important finding as it provides novel insight into high-level action representation of manipulation and the flexibility with which it can be executed.

\section{Control mechanisms}

We had proposed a control mechanism that integrates online feedback of digit placement with sensorimotor memories of the manipulation task for compensating the variance in digit placement through digit force modulation to achieve stable behavioral performance ( $\mathrm{Fu}$ et al., 2010). This compensation is implemented according to a high-level representation of the learned manipulation task before object lift onset. However, in that study force compensation for trial-to-trial variability of digit placement was implemented on the digits that had already experienced the manipulation task (e.g., same degrees of freedom). It is remarkable that this compensation could occur even when adding or removing a digit significantly changes the sensory feedback (e.g., activation of cutaneous receptors, proprioceptors) relative to that associated with the manipulation learned before the switch in grip type. Within this framework, the force compensation for trial-to-trial variability in digit placement described by $\mathrm{Fu}$ et al. (2010) appears to be a special case of the general phenomenon revealed by the present study. The proposed concept of decomposing high-level representation into different degrees of freedom (digit positions and forces) is similar to the virtual finger hypothesis (Iberall et al., 1986; Baud-Bovy and Soechting, 2001), which argues that prehension is planned first using virtual fingers in opposition space, and then mapped into individual fingers. However, if the position of the virtual finger were to be identified with the location of the CoP, our results do not support an invariant virtual finger in different grips. The manipulation task could have been directly mapped into individual digits without necessarily involving virtual fingers.

\section{Cortical networks required for within-hand transfer of learning in manipulation}

Within-hand transfer of dexterous manipulation requires the following three neural processes: (1) generation of high-level representation (i.e., net force/torque applied on the object) derived from the integration of feedback sensed through arbitrary sensory elements (i.e., digit contact distribution and forces); (2) storage, update, and retrieval of the high-level representation of the task; and (3) effective decomposition into arbitrary degrees of freedom.

Posterior parietal cortex (PPC) receives sensory signals from different sensory modalities as well as efferent copies from motor cortex (Andersen et al., 1997). Brain imaging studies indicate that PPC is involved in the coordination of fingertip forces (Ehrsson et al., 2003; Jenmalm et al., 2006) and sensorimotor transformations (Jeannerod et al., 1995; Avillac et al., 2005). In our task, building a given high-level representation of manipulation means integrating sensory feedback of digit forces and positions from an arbitrary set of digits. We propose that this area is involved in transforming digit-specific force vectors and relative positions into a neural representation of the net torque exerted on the object.

Neural representation of the net force/torque necessary for manipulation (in our task, the compensatory torque to prevent object roll during the lift) needs to be accurately stored and retrieved. 
Rijntjes et al. (1999) showed that end-effector-independent functional representations of signing movements performed with fingers and toes appear to be stored in, and retrieved from, the same cortical network involving secondary sensorimotor cortices (i.e., the anterior part of ventral premotor and dorsal cortices, supplementary motor area, middle and ventral intraparietal areas in the intraparietal sulcus, thalamus, and cerebellar hemispheres). Therefore, this network could be involved in storing a neural representation of the "goal" of the task (i.e., compensatory torque), as opposed to effector-specific areas (e.g., primary sensorimotor cortex) that are selectively involved when using muscles involved with different grip types.

We propose that the decomposition of the high-level representation into neural commands to degrees of freedom (e.g., muscles of the thumb, index, and middle finger) occurs at the planning stage and is further refined through somatosensory feedback from contact to object lift onset. Before contact, planning of digit forces and positions engages a frontal-parietal circuit comprised of anterior intraparietal sulcus and ventral premotor cortex (Davare et al., 2007; Olivier et al., 2007). After contact, we speculate that the same cortical networks are involved for digit position sensing and are used to modulate forces if unexpected deviations from desired contact positions are detected (Fu et al., 2010). Jenmalm et al. (2006) have shown that supramarginal gyrus may monitor the mismatch between predicted and actual sensory input and update sensorimotor memories in a lifting task, whereas corrections to erroneously programmed lifting force involved supplementary motor area and cerebellum. It is likely that monitoring the compensatory torque in our task may engage the same neural circuitry, but further studies are needed to test this model.

The proposed circuitry underlying the learning of object manipulation (Fu et al., 2010) and the above-described neural networks underlying motor equivalence are likely to significantly overlap. Such overlap is mostly determined by the fact that both learning and learning transfer require the ability to generate and retrieve a high-level representation of the task that is not constrained by a specific digit contact distribution. Therefore, learning manipulation using the same digits may become indistinguishable from transferring learned manipulation involving a variable number of digits, the main differences being how accurate the stored prior high-level representation is and the extent to which it needs to be updated.

\section{Are learned manipulations always transferable?}

The seamless learning transfer of our manipulation task across grip types raises the following question: is the ability of transferring learned manipulations a fundamental ability of the CNS or, conversely, is it highly dependent on the conditions of the task subjects transfer the learned manipulation to? Several lines of evidence support the latter scenario. Specifically, subjects are unable to fully transfer manipulative forces following an object rotation that changes the learned mapping between digit forces and object properties, for example, texture (Edin et al., 1992; Quaney and Cole, 2004) or mass distribution (Salimi et al., 2000, 2003; Bursztyn and Flanagan, 2008; Albert et al., 2009; Ingram et al., 2010; Zhang et al., 2010).

The above studies suggest that the CNS might store multiple neural representations of manipulation tasks (Ingram et al., 2010). Yet, the present findings suggest the existence of a highlevel, effector-independent representation of learned manipulations that can be easily transferred. However, the studies that have described the failure of learning transfer all required subjects to successfully dissociate the frame of reference of the learned manipulation from a hand-centered frame of reference, whereas the present study did not. Therefore, we speculate that anticipatory control of the compensatory torque did not degrade despite changing grasp configuration because the frame of reference of the manipulation task remained invariant relative to the hand frame of reference.

Interestingly, however, there are instances where the congruence between manipulation task and hand frame of reference can be broken by changing the hand position relative to the object without interfering with transfer of learned manipulation (Quaney and Cole, 2004; Bursztyn and Flanagan, 2008). These two studies, together with the above-cited work, suggest that the interference to learning transfer is not caused by the lack of congruence between hand and manipulation frames of reference per se, but rather by the inability of the CNS to mentally rotate the action as a function of the object's new orientation relative to the hand.

\section{References}

Albert F, Santello M, Gordon AM (2009) Sensorimotor memory of object weight distribution during multidigit grasp. Neurosci Lett 463:188-193.

Andersen RA, Snyder LH, Bradley DC, Xing J (1997) Multimodal representation of space in the posterior parietal cortex and its use in planning movements. Annu Rev Neurosci 20:303-330.

Avillac M, Denève S, Olivier E, Pouget A, Duhamel JR (2005) Reference frames for representing visual and tactile locations in parietal cortex. Nat Neurosci 8:941-949.

Baud-Bovy G, Soechting JF (2001) Two virtual fingers in the control of the tripod grasp. J Neurophysiol 86:604-615.

Bernstein N (1967) The co-ordination and regulation of movements. Oxford, UK: Pergamon.

Budgeon MK, Latash ML, Zatsiorsky VM (2008) Digit force adjustments during finger addition/removal in multi-digit prehension. Exp Brain Res 189:345-359.

Bursztyn LL, Flanagan JR (2008) Sensorimotor memory of weight asymmetry in object manipulation. Exp Brain Res 184:127-133.

Chang EC, Flanagan JR, Goodale MA (2008) The intermanual transfer of anticipatory force control in precision grip lifting is not influenced by the perception of weight. Exp Brain Res 185:319-329.

Cole KJ, Abbs JH (1986) Coordination of three-joint digit movements for rapid finger-thumb grasp. J Neurophysiol 55:1407-1423.

Davare M, Andres M, Clerget E, Thonnard JL, Olivier E (2007) Temporal dissociation between hand shaping and grip force scaling in the anterior intraparietal area. J Neurosci 27:3974-3980.

Edin BB, Westling G, Johansson RS (1992) Independent control of human finger-tip forces at individual digits during precision lifting. J Physiol 450:547-564.

Ehrsson HH, Fagergren A, Johansson RS, Forssberg H (2003) Evidence for the involvement of the posterior parietal cortex in coordination of fingertip forces for grasp stability in manipulation. J Neurophysiol 90:2978-2986.

Fu Q, Zhang W, Santello M (2010) Anticipatory planning and control of grasp positions and forces for dexterous two-digit manipulation. J Neurosci 30:9117-9126.

Goodwin AW, Jenmalm P, Johansson RS (1998) Control of grip force when tilting objects: effect of curvature of grasped surfaces and applied tangential torque. J Neurosci 18:10724-10734.

Iberall T, Bingham G, Arbib MA (1986) Opposition space as a structuring concept for the analysis of skilled hand movements. In: Generation and modulation of action patterns (Heuer H, Fromm C, eds), pp 158-173. Berlin: Springer-Verlag.

Ingram JN, Howard IS, Flanagan JR, Wolpert DM (2010) Multiple graspspecific representations of tool dynamics mediate skillful manipulation. Curr Biol 20:618-623.

Jeannerod M, Arbib MA, Rizzolatti G, Sakata H (1995) Grasping objects: the cortical mechanisms of visuomotor transformation. Trends Neurosci 18:314-320.

Jenmalm P, Schmitz C, Forssberg H, Ehrsson HH (2006) Lighter or heavier than predicted: neural correlates of corrective mechanisms during erroneously programmed lifts. J Neurosci 26:9015-9021. 
Jesunathadas M, Zhang W, Santello M (2010) Learning and transfer across hands of skilled object manipulation. Soc Neurosci Abstr 36:789.4/WW16.

Lashley K (1930) Basic neural mechanisms in behavior. Psychol Rev $37: 1-24$.

Lukos J, Ansuini C, Santello M (2007) Choice of contact points during multidigit grasping: effect of predictability of object center of mass location. J Neurosci 27:3894-3903.

Lukos JR, Ansuini C, Santello M (2008) Anticipatory control of grasping: independence of sensorimotor memories for kinematics and kinetics. J Neurosci 28:12765-12774.

Olivier E, Davare M, Andres M, Fadiga L (2007) Precision grasping in humans: from motor control to cognition. Curr Opin Neurobiol 17:644-648.

Quaney BM, Cole KJ (2004) Distributing vertical forces between the digits during gripping and lifting: the effects of rotating the hand versus rotating the object. Exp Brain Res 155:145-155.
Rijntjes M, Dettmers C, Büchel C, Kiebel S, Frackowiak RS, Weiller C (1999) A blueprint for movement: functional and anatomical representations in the human motor system. J Neurosci 19:8043-8048.

Salimi I, Hollender I, Frazier W, Gordon AM (2000) Specificity of internal representations underlying grasping. J Neurophysiol 84:23902397.

Salimi I, Frazier W, Reilmann R, Gordon AM (2003) Selective use of visual information signaling objects' center of mass for anticipatory control of manipulative fingertip forces. Exp Brain Res 150:9-18.

Santello M, Soechting JF (2000) Force synergies for multifingered grasping. Exp Brain Res 133:457-467.

Wing AM (2000) Motor control: mechanisms of motor equivalence in handwriting. Curr Biol 10:245-248.

Zhang W, Gordon AM, Fu Q, Santello M (2010) Manipulation after object rotation reveals independent sensorimotor memory representations of digit positions and forces. J Neurophysiol 103:2953-2964. 